Retos, II (2I), 202 I

\title{
La empresa española ante la COVID-19: factores de adaptación al nuevo escenario
}

\section{The Spanish company in the face of COVID-19: adaptation factors to the new scenario}

Mg. Ricardo Diego Pérez-Calle es profesor e investigador de la Universidad de Zaragoza (España) (rdperez@unizar.es) (https://orcid.org/0000-0003-2726-7146)

Dra. Nieves García-Casarejos es profesora e investigadora de la Universidad de Zaragoza (España) (ngarcia@unizar.es) (https://orcid.org/0000-0002-5142-0270)

Dr. Javier García-Bernal es profesor e investigador de la Universidad de Zaragoza (España) (igbernal@unizar.es) (https://orcid.org/0000-0002-4540-4952)

\begin{abstract}
Resumen
La pandemia de la COVID-19 ha cambiado de forma radical e impredecible el entorno competitivo de las empresas, siendo España uno de los países de su entorno que se ha visto más afectado. En este nuevo contexto, para hacer frente a unas condiciones del entorno extremadamente dinámicas, complejas y difícilmente predecibles, las empresas para poder adaptarse necesitan estar en posesión o desarrollar una serie de capacidades específicas. Este artículo pretende mostrar que el desarrollo de las TIC, la flexibilidad laboral y la capacidad de innovar son tres factores que favorecen la capacidad de las organizaciones para adaptarse a los nuevos y cambiantes entornos competitivos provocados por la emergencia sanitaria de la COVID-19. La capacidad de adaptarse se refleja a través de su habilidad para dar continuidad tanto a la gestión y monitorización de sus operaciones como a la relación con sus clientes a lo largo de todos los niveles del proceso de venta. Para analizar esta relación, se entrevistó a directivos de empresas durante los meses posteriores a la declaración del estado de alarma en España. Mediante la aplicación de la técnica de análisis multivariante PLS-SEM, se ha estimado que un mayor nivel de los tres aspectos indicados se relaciona positivamente con una mejor adaptación de las empresas tanto a las nuevas necesidades como a las limitaciones de operación en sus respectivos mercados.
\end{abstract}

\begin{abstract}
The COVID-19 pandemic has radically and unpredictably changed the competitive environment for companies, with Spain being one of the western countries most affected. In this new context, to face extremely dynamic, complex, and hardly predictable competitive environments, companies must adapt by having or developing a series of specific resources and capacities. This article aims to show that the development of Information and Communication Technology, labor flexibility and the capacity to innovate are factors that favor organizations having the ability to adapt to the new and changing competitive environments caused by the health emergency of COVID-19, reflecting the organization's ability to adapt through its ability to give continuity to both the management and monitoring of its operations and the relationship with its customers throughout all levels of the sales process. To analyze this relationship, company managers were interviewed during the months after the declaration of the state of alarm in Spain. Through the application of the multivariate analysis technique Partial Least Squares Structural Equation Modeling (PLS-SEM), it has been estimated that high levels of ICT development, labor flexibility or capacity to innovate are positively related to a better adaptation of companies to both the new needs of their customers as well as the operating limitations imposed in their respective markets.
\end{abstract}

\section{Palabras clave I keywords}

Gestión, TIC, flexibilidad, innovación, COVID-19, PLS-SEM, capacidad de adaptación, entorno competitivo. Management, ICT, flexibility, innovation, COVID-19, PLS-SEM, adaptability, competitive environment.

Cómo citar: Pérez-Calle, R.D., García-Casarejos, N., y García-Bernal, J. (2021). La empresa española ante la COVID-19: factores de adaptación al nuevo escenario. Retos Revista de Ciencias de la Administración y Economía, 11(21), pp. 5-24. https://doi.org/10.17163/ret.n21.2021.01 


\section{Introducción}

La pandemia de la COVID-19, desde su declaración a finales de 2019, ha impuesto una transformación radical en el comportamiento de los distintos agentes económicos a nivel global. En concreto, el impacto que ha ejercido sobre la actividad económica española ha sido devastador, convirtiendo al país en uno de los más afectados de su entorno. Si al final del tercer trimestre de 2020 la caída del PIB en la zona euro, respecto a los niveles de antes de la crisis, era del 4,5\%, en España fue del 9,1\%. Las previsiones de la Organización para la Cooperación y el Desarrollo Económicos (OCDE) para el conjunto del año 2020 son de una caída del 11,6\%, la mayor de las de todos los países de la organización (OECD, 2021). Y dichas previsiones establecen la recuperación de los niveles anteriores a la crisis para el segundo semestre de 2022. La sucesión de olas de contagios y las consecuentes restricciones para la actividad económica o la movilidad no hacen prever un escenario más optimista.

Desde la declaración del estado de alarma en España en marzo de 2020, miles de empresas y trabajadores han visto frenada o suspendida su actividad. En concreto, según el informe presentado por el Banco de España en diciembre de 2020 (Blanco et al., 2020), se estima que hasta el 9,9\% de las empresas españolas han podido desaparecer por problemas de solvencia motivados por la crisis generada por la COVID-19. Esto supone un 150\% de incremento respecto a los datos de 2019. Un indicador de esta situación que ofrece el informe es que el 40,6\% de las empresas tienen una presión financiera elevada, frente al 13,9\% que la tenían el año anterior. Estos datos se explican teniendo en cuenta que durante los primeros nueve meses del año, el 36\% de las empresas españolas han tenido pérdidas en su cuenta de resultados. Y todo ello a pesar de que la situación de las empresas españolas antes de la pandemia era, en términos medios, de fortaleza financiera.

No todos los sectores económicos se han visto afectado por igual. El confinamiento y las restricciones de movilidad y de reunión han afectado a las condiciones de oferta y demanda de forma muy heterogénea en los distintos mercados, así como a las relaciones entre las distintas cadenas y sistemas de valor. Se calcula que, en los sectores de hostelería, restauración y ocio, un $72,4 \%$ de las empresas tendrá problemas de viabilidad por incapacidad para pagar sus deudas. En el de vehículos de motor este problema se estima que lo sufrirán el 64,6\% de las compañías, el 42,2\% de las del sector de comercio y el 41,6\% de las dedicadas al transporte y almacenaje (Blanco et al., 2020).

El comportamiento de las organizaciones en entornos de alta volatilidad provocados por shocks exógenos ha sido ampliamente estudiado en la literatura, bajo distintas denominaciones: sucesos no esperados, eventos raros, crisis o catástrofes (Duchek et al., 2020). No obstante, el origen de esta situación social y económica, la pandemia de la COVID-19, constituye un evento que se ajusta con todas las características propias de un suceso "cisne negro" (Taleb, 2008): un suceso sorpresivo impredecible, de gran impacto socioeconómico y que se puede racionalizar por retrospección (apariencia predecible posterior). De esta forma, las implicaciones y consecuencias de un suceso de estas características suponen un impacto mayor que las de los sucesos predecibles, efectos más duraderos y con un fuerte componente transversal.

En este contexto factual, los análisis sobre los factores de adaptación a entornos volátiles, dinámicos y complejos, pero en mayor o menor medida predecibles, dejan de ser válidos, dado que las condiciones impuestas por la emergencia sanitaria han cambiado radicalmente, y de forma literal de la noche a la mañana, las condiciones de operación de las empresas. Y lo continúan haciendo. La sucesión de oleadas de contagios ha provocado que las restricciones a la actividad económica y libertad de movi- 
miento se hayan ido a su vez sucediendo, por lo que las empresas han tenido que seguir adaptando sus actividades a cambios radicales de su entorno competitivo. De todo ello se puede extraer la conclusión de que las empresas que estuvieran en posesión de los recursos y capacidades necesarios para una rápida adaptación reactiva a los sucesivos y cambiantes entornos competitivos han podido afrontar dichas nuevas condiciones impuestas en una situación de ventaja competitiva frente a las que no estuvieran en posesión de dichos recursos y capacidades (Barney, 2001).

El objetivo de la presente investigación es explorar, en el caso español, posibles respuestas a la pregunta de qué factores determinan que las empresas estén dotadas de la capacidad de adaptación necesaria a las nuevas y cambiantes condiciones del entorno competitivo provocadas por la pandemia, sin entrar a analizar las particularidades de las empresas y la economía española que han hecho que la intensidad de la crisis consecuencia de la pandemia haya sido mayor que en el resto de países de su entorno.

Se han considerado tres factores para analizar esta relación: la capacidad de innovación de las organizaciones, su flexibilidad laboral y su nivel de desarrollo e implantación de las Tecnologías de Información y Comunicación. Estas tres variables, como se expondrá más adelante, son ampliamente consideradas como propulsoras o como dimensiones de los atributos relacionados con una capacidad de adaptación activa y con la resiliencia organizacional en momentos de crisis: rapidez, agilidad, capacidad de aprendizaje, capacidad de reinvención (Alday et al., 2020; Duchek, 2020; Freije \& Aláez, 2020; Garamendi, 2020; Weick \& Sutcliffe, 2001).

El trabajo se ha organizado como sigue. En primer lugar, se exponen las bases teóricas y las hipótesis a contrastar. A continuación, se describe la metodología empleada en la investigación, incluyendo la toma de datos muestral y la metodología de análisis multivariante empleada. Después se presentan y analizan los resultados obtenidos en el modelo estimado con los criterios de validación aplicados. Para finalizar, se realiza una discusión de los resultados obtenidos y exposición de las conclusiones extraídas, así como establecimiento de los próximos pasos a realizar en línea con la investigación.

\subsection{Marco teórico e hipótesis}

\subsubsection{La capacidad de adaptación ante la crisis}

La pandemia de la COVID-19 ha exigido a las empresas adaptar, de la forma más rápida y eficiente posible, sus estructuras, actividades, productos o procesos a las nuevas condiciones competitivas. Distintos estudios (Duchek, 2020; Hermann, 1963; Pearson \& Clair, 1998; Smart \& Vertinsky, 1984; Lengnick-Hall et al., 2011) analizan la capacidad de adaptación de las organizaciones ante situaciones de crisis inesperadas con alto potencial de consecuencias. Factores comunes considerados en ellos son la actitud proactiva, la continua observación del entorno o la habilidad de reconstruirse y reinventarse. Sin embargo, las características ya descritas del shock exógeno producido por la COVID-19 hacen que los determinantes de la capacidad de adaptación varíen frente a situaciones más previsibles o de evolución menos dinámica o traumática. En este contexto, los análisis sobre cómo afrontar con éxito la crisis se centran en conceptos difícilmente cuantificables como la resiliencia empresarial (Garamendi, 2020), la sostenibilidad (Schaltegger, 2020) o la explotación de opciones estratégicas como la coopetition (Crick \& Crick, 2020).

Esta investigación pretende determinar algunos de los factores que favorecen sortear con éxito el actual entorno competitivo. Así, se ha definido como variable endógena la "capacidad de adaptación" de las organizaciones, considerada como la capacidad de estar en sincronía con los sucesivos entornos competitivos, la rapidez en hacerlo y 
la eficiencia del proceso (Grant, 2014; Hax \& Majluf, 2015; Thompson \& Strickland, 2004). Dicho ajuste de la empresa a las nuevas condiciones externas implica que la empresa deberá alinear aspectos relacionados con:

- Necesidades de recursos.

- Oferta de productos /servicios, tanto mix como cantidad demandada.

- Apalancamiento operativo.

- Procedimientos operativos.

- Relación con stakeholders externos, especialmente clientes.

\subsubsection{La relación entre el desarrollo de las TIC y la capacidad de adaptación}

Las Tecnologías de Información y Comunicación (TIC) constituyen un campo heterogéneo y del que existen multitud de definiciones y clasificaciones. Agrupan un conjunto de recursos e innovaciones de hardware, software, telecomunicaciones o dispositivos electrónicos vinculados entre sí, con capacidad de generar entre todos ellos una red universal o herramientas que permiten acumular datos para sintetizar y generar información. Constituyen por tanto un conjunto de medios, herramientas y dispositivos que emplean tanto las telecomunicaciones como las tecnologías de computación para generar y transmitir información (Cobo-Romaní, 2009).

Respecto a la dimensión organizativa y empresarial de las TIC, se identifica con el conjunto de recursos que proporciona la capacidad de manipular información y que soportan el desarrollo y crecimiento económico de cualquier organización (Thompson \& Strickland, 2004). En todo caso, las TIC proporcionan a las organizaciones enormes posibilidades y diferentes enfoques para gestionar su conocimiento, aumentar su flexibilidad, potenciar la interactividad con sus stakeholders, favorecer su robustez financiera, aumentar su rapidez de desarrollo, favorecer su independencia, etc.

A pesar del consenso entre los académicos de que las TIC constituyen un factor que favorece significativamente el crecimiento y las posibilidades de supervivencia de las empresas, los mecanismos concretos que subyacen a dichas consecuencias han sido objeto de amplio debate (Bharadwaj, 2000), si bien existe cierto consenso sobre uno de sus efectos más valorados: el de potenciador y soporte de las competencias básicas de la organización (Ravichandran \& Lertwongsatien, 2005).

Así, directa o indirectamente, el nivel de desarrollo de las TIC o de digitalización de las organizaciones tiene una incidencia clave y un efecto permanente en los procesos, el comportamiento, el rendimiento o la cultura organizacionales (Bloom et al., 2014; Draca et al., 2007).

Por lo tanto, basándonos en ello formulamos la siguiente hipótesis:

H1: Existe una relación positiva y significativa entre el desarrollo de TIC de una empresa y su capacidad de adaptación ante el shock externo producido por la COVID-19.

\subsubsection{La relación entre el esfuerzo en innovación y la capacidad de adaptación}

La innovación se ha convertido durante las últimas décadas en un elemento fundamental para la supervivencia de las empresas, cuando anteriormente había sido considerada como elemento diferenciador. Y en el entorno turbulento marcado por la COVID-19, el factor innovación de las empresas está siendo percibido como catalizador de la gestión eficiente de las organizaciones y como generador de oportunidades a múltiples niveles de su cadena de valor (Guderian et al., 2020; Lee \& Trimi, 2020; Sharma et al., 2020). 
La innovación tiene distintos determinantes, siendo el fundamental la capacidad de innovación (Prajogo \& Ahmed, 2006), considerada como la capacidad de la empresa para generar nuevo conocimiento que será posteriormente aplicado de forma práctica en la misma, ya sea a través de la oferta de nuevos productos o servicios o a través del desarrollo de nuevos procesos. A su vez, la capacidad de innovación se puede descomponer en esfuerzo y experiencia en innovar (López-Mielgo et al., 2012). En nuestra investigación se ha considerado el primer componente, ya que este determina de forma significativa del segundo, no quedando además sesgado o moderado por la antigüedad de la organización.

Así, el esfuerzo en innovación de la organización se identifica con la búsqueda de generación de conocimiento nuevo, para su posterior aplicación en nuevos productos o servicios que ofrecer en el mercado o en nuevos procesos internos, estructuras organizativas, etc., Su medición se realiza a través de las actividades en I+D+i implementadas (Adler \& Shenbar, 1990; Guan \& Ma, 2003).

Con base en todo ello formulamos la siguiente hipótesis:

H2: Existe una relación positiva y significativa entre el esfuerzo en innovación de una empresa y su capacidad de adaptación ante el shock externo producido por la COVID-19.

\subsubsection{La relación entre la flexibilidad y la capacidad de adaptación}

La flexibilidad organizativa representa la habilidad para adaptarse de forma rápida y eficiente a un entorno incierto — en cuanto a las condiciones de la demanda y del mercado-aplicando una combinación de recursos y capacidades (Bueno, 2007; Volberda, 1996).

La flexibilidad de la organización puede dividirse en tres dimensiones (Sánchez et al., 2011):

- Flexibilidad productiva: capacidad para alcanzar la adecuada versatilidad en volumen y tipología de productos o servicios ofertados.

- Flexibilidad de la estructura organizativa: capacidad de adecuación a nivel de estructura jerárquica, descentralización, tamaño, internacionalización o integración vertical.

- Flexibilidad laboral: capacidad de los recursos humanos de adaptarse a las condiciones internas y externas de la organización.

La presente investigación se ha enfocado en la flexibilidad laboral, considerada como facilitadora de las flexibilidades organizativas y productivas (Wright \& Snell, 1998). Atkinson (1981) establece la siguiente tipología de flexibilidad laboral: numérica, financiera y funcional. Las dos primeras han quedado excluidas del estudio, ya que la primera hace referencia a la capacidad de adaptar el tamaño de la fuerza laboral a la demanda — situación que las empresas han realizado mediante las figuras legales de ERE y ERTE_ - y la segunda a las condiciones retributivas - también determinadas por distintas leyes y regulaciones. La tercera se refiere a la polivalencia o autonomía de los empleados como factor de adaptación de la fuerza laboral a las exigencias del mercado. En este contexto, es importante resaltar la relación establecida entre la resiliencia organizacional y la gestión de los recursos humanos, en la que se identifica que la primera se ve favorecida por el nivel de competencias en posesión de los empleados (Lengnick-Hall et al., 2011).

De esta forma, formulamos la siguiente hipótesis: 
H3: Existe una relación positiva y significativa entre la flexibilidad laboral de una organización y su capacidad de adaptación ante el shock externo producido por la COVID-19.

\subsubsection{El papel mediador del esfuerzo en innovación}

La influencia positiva que la flexibilidad ejerce sobre la capacidad de adaptación puede analizarse también a través de la influencia que la primera ejerce sobre el esfuerzo en innovación. Dicha influencia, a nivel de la flexibilidad laboral funcional, ha sido analizada a través de diversos canales que favorecen la capacidad de innovación (Sánchez et al., 2011):

- La polivalencia mejora las habilidades individuales.

- La polivalencia favorece el trabajo en equipo y la colaboración interdepartamental.

- La polivalencia amplía la difusión del conocimiento.

- La polivalencia mejora la motivación por eliminación del trabajo repetitivo

- La formación en el puesto de trabajo desarrolla a los empleados del núcleo competitivo de la organización.

Además, la capacidad de innovación de las empresas influye y potencia de forma transversal las capacidades de la organización y su relación con los distintos stakeholders (López-Mielgo et al., 2012).

Todo lo anterior nos lleva a formular la siguiente hipótesis:

H4: Existe una relación positiva y significativa entre la flexibilidad de una organización y su capacidad de adaptación ante la COVID-19, a través del canal del esfuerzo en innovación.

\subsubsection{Modelo teórico}

Con los recursos, fundamentos y razonamientos expuestos, planteamos el siguiente modelo teórico.

\section{Figura 1. Modelo teórico}

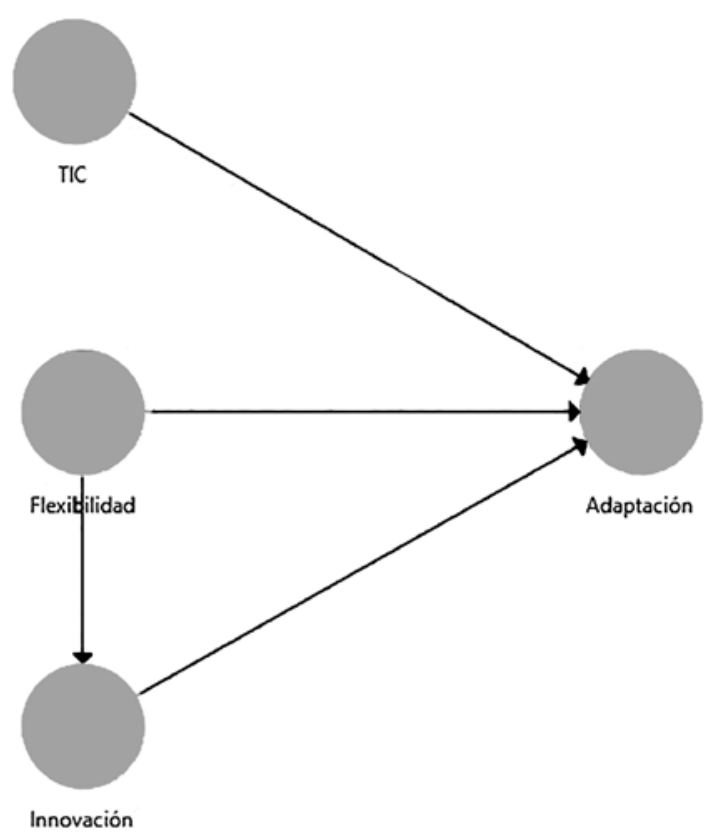

Fuente: Autores. 


\section{Materiales y método}

El análisis exploratorio de nuestro modelo teórico se ha realizado mediante Modelización de Ecuaciones Estructurales con Mínimos Cuadrados Parciales (PLSSEM), a través de la aplicación de software SmartPLS 3.3.2. Los motivos de esta elección fueron su adecuación al carácter exploratorio de la investigación, su flexibilidad para el uso simultáneo de indicadores formativos y reflectivos y de diferentes escalas de medición, así como su capacidad para emplear variables no paramétricas.

La toma de datos se llevó a cabo mediante una encuesta realizada a 76 directivos de empresas entre los meses de mayo a agosto de 2020. El tamaño medio de las empresas incluidas en la muestra fue de 527 trabajadores, con una mediana de 60, lo cual se acerca a la representación del tejido económico español si se obvian las empresas individuales. No se incluyeron en la muestra empresas de los sectores más afectados por la pandemia y las restricciones derivadas, como hostelería y restauración, turismo, transportes, ocio, automoción o determinado comercio minorista (Blanco et al., 2020).

La encuesta se construyó con preguntas abiertas sobre distintas actividades, prácticas o recursos relacionados con las variables de nuestro modelo teórico y con las dificultades surgidas tras el estado de alarma. Para la observación y medición a través de la encuesta de las cuatro variables latentes del modelo se definieron un conjunto de indicadores o variables manifiestas.

Variable latente "TIC": constructo con un indicador formativo, Digi, cuantificado por escala Likert de 1 a 3, tomando como base las clasificaciones por áreas temáticas y niveles de desarrollo de las TIC establecidas por el Ministerio de Industria, Comercio y Turismo del Gobierno de España (Observatorio Nacional de las Telecomunicaciones y de la Sociedad de la Información, 2011, 2020):

- Nivel 1 (Likert 1): infraestructura tecnológica básica (ordenador, servidores, etc.); infraestructura de comunicaciones básica (telefonía, internet).

- Nivel 2 (Likert 2): soluciones de ámbito general (ofimática, ERP, etc.). Perfiles en RRSS. Desarrollo de página web o comercio electrónico.

- Nivel 3 (Likert 3): industria 4.0, servicios TIC avanzados (herramientas específicas para procesos de negocio, administración electrónica, información en tiempo real, publicidad dirigida, Big Data, etc.).

Variable latente "Innovación": compuesto de un indicador formativo, IDi. Las actividades en $\mathrm{I}+\mathrm{D}+\mathrm{i}$ se consideran primer paso y propulsoras de la innovación (Cohen \& Levinthal, 1990; Janowski, 1998; López-Mielgo et al., 2012), por lo que se tomó a los recursos dedicados a $\mathrm{I}+\mathrm{D}+\mathrm{i}$ como proxy de este constructo. La cuantificación de las observaciones realizadas a través de la encuesta del indicador se ha realizado, mediante escala Likert de 1 a 3, con base al ajuste de las respuestas obtenidas respecto a los recursos dedicados a las actividades de $\mathrm{I}+\mathrm{D}+\mathrm{i}$ con el siguiente patrón:

- No se realizan o se realizan como un esfuerzo extraordinario.

- Se realizan actividades de forma habitual. Sin presupuesto comprometido/ estructura formal para su realización.

- El esfuerzo en $\mathrm{I}+\mathrm{D}+\mathrm{i}$ es parte del funcionamiento normal de la empresa, con porcentaje establecido del presupuesto anual y personas/departamento/división responsable. 
Variable latente "Flexibilidad": compuesto con dos constructos formativos, Aut y Multi. Considerándose la flexibilidad laboral funcional (Atkinson, 1981), las dos dimensiones observadas fueron la redistribución de los trabajadores con base en su polivalencia (indicador Multi) y la autonomía del empleado en la realización del trabajo (Aut). La cuantificación de cada indicador fue por escala Likert de 1 a 5, aplicando los criterios expuestos por Bloom y Van Reenen (2007):

Cuantificación del indicador Aut: "el ritmo de trabajo y tareas desempeñadas por los trabajadores en el día a día lo deciden”:

1: Empresario/directivo todas.

2: Empresario/directivo la mayoría.

3: Consenso/equilibrio.

4: Trabajador la mayoría.

5: Trabajador todas.

Cuantificación del indicador Multi: "en la organización existe":

1: Alta especialización de los trabajadores para lograr máxima eficiencia. No existe movilidad entre puestos.

2: Cierta movilidad ad hoc cuando las necesidades de producción lo exigen.

3: Movimientos formales entre puestos por necesidades de producción. No hay programa específico de desarrollo de la polivalencia del trabajador.

4: Movimientos formales entre puestos por necesidades de producción. Existe un programa específico de desarrollo de la polivalencia del trabajador.

5: Fomento y desarrollo de la polivalencia de los trabajadores. La rotación es parte del funcionamiento operativo normal de la empresa.

Variable latente "Adaptación”: medida por cinco indicadores reflectivos, Costes, P/S, Pers, Org, RelCl. Cada uno se corresponde con efectos que una mayor o menor capacidad de adaptación al entorno provoca en las organizaciones. La observación de cada uno se ha realizado a través de las siguientes preguntas y la cuantificación de las observaciones con base en las correspondientes respuestas obtenidas:

- Costes: “¿La situación provocada por la COVID-19 ha forzado a su empresa a reducir costes de estructura (excluidos los de personal) para reducir el apalancamiento operativo de la empresa?". Cuantificación por Likert de 1 (mucho) a 3 (poco/nada).

- Pers: “¿La situación provocada por la COVID-19 ha forzado a su empresa a variar de forma continua el número de trabajadores en plantilla?”. Likert de 1 (mucho) a 3 (poco/nada).

- P/S: “¿La situación provocada por la COVID-19 ha forzado a su empresa, por problemas operativos, a variar el mix de productos/servicios ofertados?”. Likert de 1 (mucho) a 3 (poco/nada).

- Org: “¿La situación de provocada por la COVID-19 ha dificultado la organización o monitorización del trabajo a nivel operativo en la empresa?”. Likert 1 a 5:

1: La organización y monitorización del trabajo se han visto muy afectadas.

3: Alguna de estas actividades se han visto afectadas de forma media o temporalmente.

5: La organización y monitorización del trabajo, en general, no se han visto afectadas. 
- Rel.Cl: “¿La situación de provocada por la COVID-19 ha dificultado la relación y gestión de los clientes a nivel preventa, venta o postventa?”. Likert 1 a 5:

1: La relación con los clientes se ha visto muy afectada/dificultada a todos los niveles.

3. La relación con los clientes se ha visto afectada a nivel significativo en alguno de dichos niveles.

5: La relación con los clientes no/apenas se ha visto afectada.

Con todo ello, nuestro modelo teórico tiene cuatro variables latentes y nueve variables manifiestas:

Tabla 1. Variables latentes e indicadores del modelo teórico

\begin{tabular}{|c|c|c|c|}
\hline $\begin{array}{l}\text { Variable } \\
\text { latente }\end{array}$ & Descripción & Índice & Descripción \\
\hline TIC & $\begin{array}{l}\text { Desarrollo de las } \\
\text { TIC. }\end{array}$ & Dig & $\begin{array}{l}\text { Nivel de desarrollo alcanzado de las } \\
\text { Tecnologías de Información y Comuni- } \\
\text { cación. }\end{array}$ \\
\hline \multirow[t]{2}{*}{ Flexibilidad } & $\begin{array}{l}\text { Flexibilidad labo- } \\
\text { ral funcional. }\end{array}$ & Aut & Nivel de autonomía de los trabajadores. \\
\hline & & Multi & Polivalencia de los empleados. \\
\hline Innovación & $\begin{array}{l}\text { Esfuerzo en } \\
\text { innovación. }\end{array}$ & IDi & Recursos dedicados a $\mathrm{I}+\mathrm{D}+\mathrm{i}$ \\
\hline \multirow{5}{*}{ Adaptación } & \multirow{5}{*}{$\begin{array}{l}\text { Capacidad de } \\
\text { adaptación. }\end{array}$} & Costes & Variación apalancamiento operativo. \\
\hline & & Pers & Variación trabajadores. \\
\hline & & $\mathrm{P} / \mathrm{S}$ & Variación mix de productos/servicios. \\
\hline & & Rel.Cl. & Afectación a la relación con los clientes \\
\hline & & Org & $\begin{array}{l}\text { Incidencia en organización y monitori- } \\
\text { zación del trabajo. }\end{array}$ \\
\hline
\end{tabular}

\section{Resultados}

\subsection{Muestra}

Para la definición del tamaño de la muestra, se empleó el criterio de las tablas de potencia de Cohen (1988). Para nuestra investigación, con un número de predictores máximo de tres, potencia de 0.8 y $\sqrt{ }$ de 0.05 , se tiene que la muestra requerida para efectos medios son 76 observaciones y para efectos grandes de 35, por lo que la muestra obtenida se considera suficiente para nuestro modelo.

La tabla 2 describe los parámetros estadísticos de las variables manifiestas de la muestra. 


\section{Tabla 2. Estadísticos descriptivos}

\begin{tabular}{|c|c|c|c|c|c|}
\cline { 2 - 6 } \multicolumn{1}{c|}{} & Media & Mediana & Desv. Est. & Kurtosis & No Observ. \\
\hline Dig & 2,158 & 2,158 & 0,779 & $-1,31$ & 76 \\
\hline Aut & 2,105 & 2,105 & 0,981 & $-0,175$ & 76 \\
\hline Multi & 2,605 & 2,605 & 1,052 & $-0,383$ & 76 \\
\hline IDi & 2,816 & 2,816 & 1,243 & $-0,936$ & 76 \\
\hline Costes & 2,092 & 2,092 & 0,652 & $-0,634$ & 76 \\
\hline Pers & 2,039 & 2,039 & 0,637 & $-0,499$ & 76 \\
\hline P/S & 2,592 & 2,592 & 0,566 & 0,119 & 76 \\
\hline Rel.Cl & 3,289 & 3,289 & 1,049 & $-0,576$ & 76 \\
\hline Org & 3,066 & 3,066 & 1,017 & $-0,626$ & 76 \\
\hline
\end{tabular}

\subsection{Modelo estimado}

El procedimiento de análisis realizado ha sido el propuesto por Hair et al. (2019). La evaluación del modelo se realizó en dos etapas: evaluación del modelo estructural y evaluación del modelo de medida. Adicionalmente, también se ha realizado la evaluación del ajuste del modelo global.

Los resultados estimados fueron los siguientes:

Figura 2. Modelo estimado (R2, coeficientes path y pesos/cargas externas)

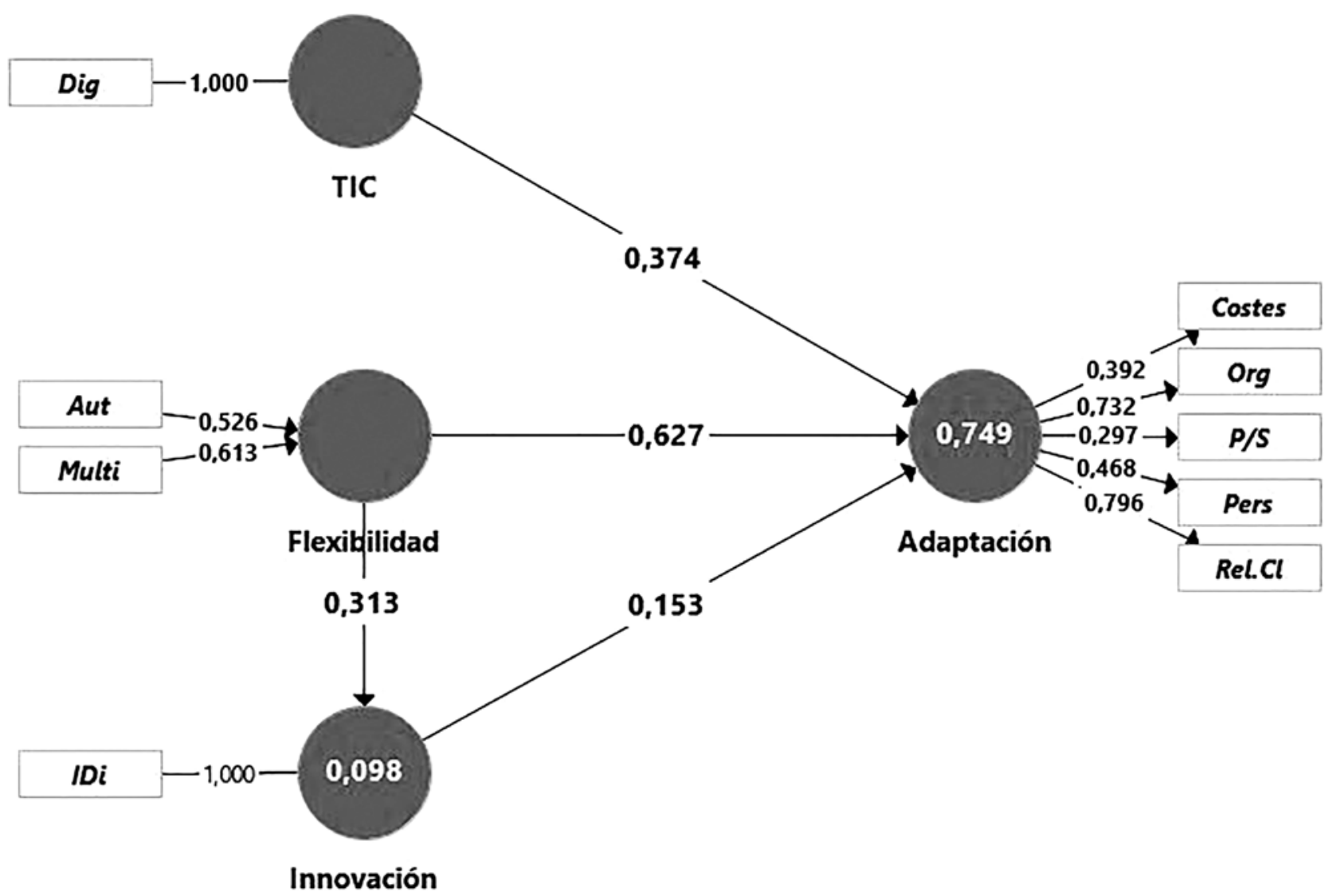

Fuente: Autores. 


\subsubsection{Evaluación del modelo de medida}

El modelo de medida muestra las relaciones entre constructos e indicadores. Mediante su análisis, se evaluó la fiabilidad y validez de los indicadores de cada constructo (Hair et al., 2019). Dada la diferente naturaleza de los modelos de medida con indicadores reflectivos y formativos la validación de cada tipo se realizó por separado. Se consideraron los siguientes criterios de validación:

Formativos (Hair et al., 2019; Chin, 2010):

- Evaluación a nivel de constructo: validez externa o convergente.

- Evaluación a nivel de indicador: valoración de existencia de multicolinealidad; valoración de la magnitud de los pesos y de su significación.

Reflectivos (Hair et al., 2019):

- Fiabilidad del indicador.

- Consistencia interna.

- Validez convergente.

- Validez discriminante.

Modelos de medida formativos ("Innovación", "TIC" y "Flexibilidad").

Análisis de la existencia de multicolinealidad: mediante el Factor de Inflación de la Varianza (VIF), donde el criterio de validación es que para valores de VIF mayores de 3,3 existe alta multicolinealidad (Diamantopoulos \& Siguaw, 2006).

Tabla 3. Factor de Inflación de la Varianza (VIF)

\begin{tabular}{|l|c|}
\cline { 2 - 2 } \multicolumn{1}{c|}{} & VIF \\
\hline IDi & 1 \\
\hline Dig & 1 \\
\hline Aut & 1,406 \\
\hline Multi & 1,406 \\
\hline
\end{tabular}

Valoración de la relevancia de los indicadores: los pesos de los distintos indicadores formativos muestran que todos ellos son relevantes en el modelo.

Tabla 4. Pesos externos

\begin{tabular}{|l|c|c|c|}
\cline { 2 - 4 } \multicolumn{1}{c|}{} & Innovación & Desarrollo TIC & Flexibilidad \\
\hline IDi & 1 & & \\
\hline Dig & & 1 & 0,526 \\
\hline Aut & & & 0,613 \\
\hline Multi & & & \\
\hline
\end{tabular}

Valoración de la significación de los indicadores: mediante el procedimiento de remuestreo bootstrapping. Los resultados muestran que los indicadores son significativos. 


\section{Tabla 5. Resultado bootstrapping para pesos externos}

\begin{tabular}{|l|c|c|c|c|c|}
\cline { 2 - 6 } \multicolumn{1}{c|}{} & $\begin{array}{c}\text { Muestra } \\
\text { original }\end{array}$ & $\begin{array}{c}\text { Media } \\
\text { muestra }\end{array}$ & $\begin{array}{c}\text { Desviación } \\
\text { estándar }\end{array}$ & Estadísticos t & P Valores \\
\hline Aut -> Flexibilidad & 0,526 & 0,504 & 0,162 & 3,25 & 0,001 \\
\hline Multi -> Flexibilidad & 0,613 & 0,625 & 0,149 & 4,106 & 0,000 \\
\hline
\end{tabular}

\section{Modelos de medida reflectivos (“Adaptación").}

Fiabilidad individual del indicador (correlación con su constructo): la carga factorial (l) ha de ser mayor que 0,707 . Se han obtenido los siguientes resultados:

\section{Tabla 6. Cargas factoriales}

\begin{tabular}{|c|c|}
\cline { 2 - 2 } \multicolumn{1}{c|}{} & $\mathbf{1}$ \\
\hline Costes & 0,392 \\
\hline Pers & 0,468 \\
\hline P/S & 0,297 \\
\hline Rel.Cl. & 0,796 \\
\hline Org & 0,732 \\
\hline
\end{tabular}

Solo los indicadores Rel.Cl y Org resultan suficientemente fiables, por lo que los otros tres se eliminaron del modelo. La estimación del modelo teórico una vez eliminados dichos tres indicadores fue la siguiente:

\section{Figura 3. Modelo estimado depurado}

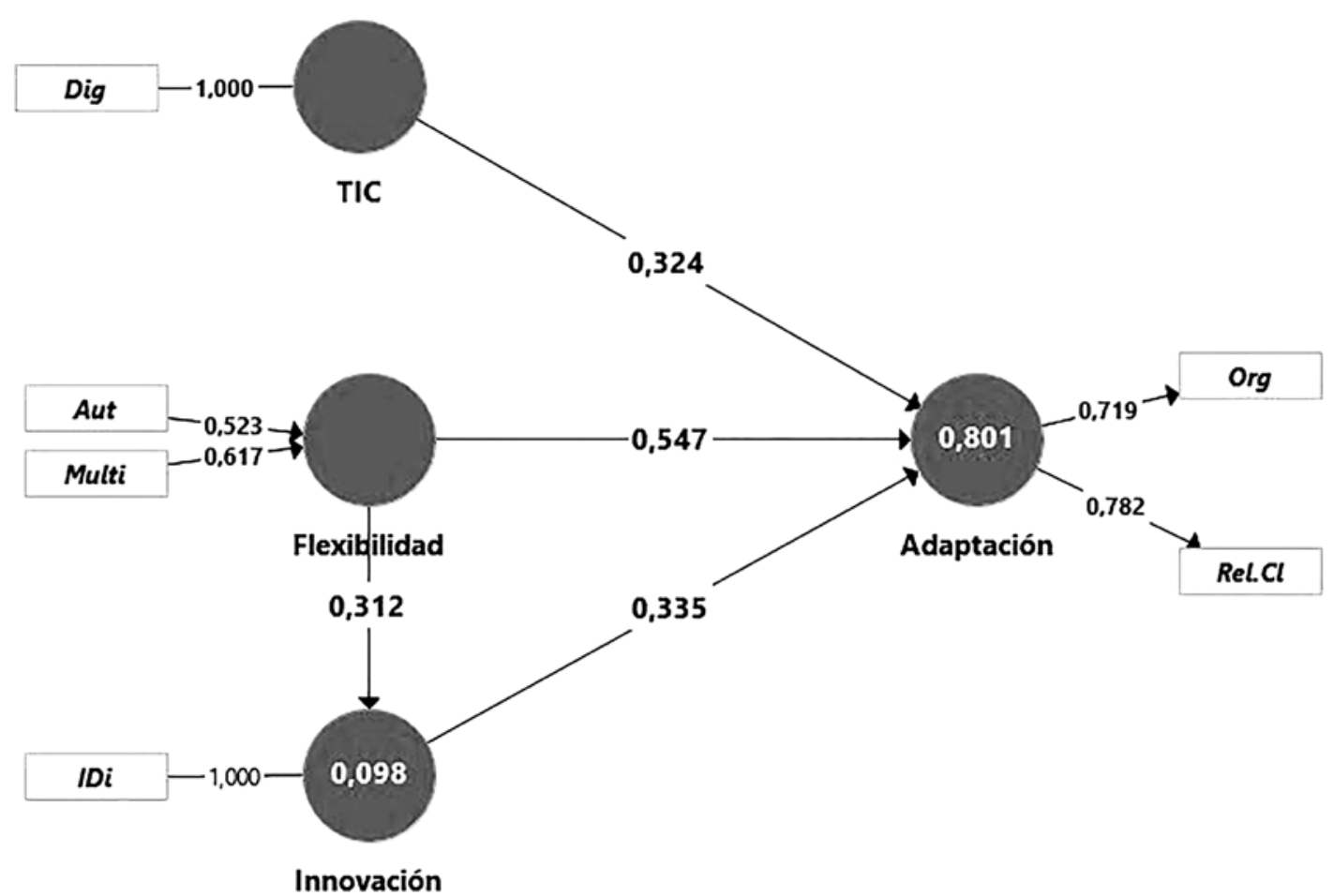

Fuente: Autores. 
Fiabilidad y validez del constructo (consistencia interna): se emplearon los siguientes criterios (Hair et al., 2019; Werts, et al., 1974; Dijkstra \& Henseler, 2015):

Tabla 7. Resultados criterios fiabilidad y validez del constructo

\begin{tabular}{|l|c|c|c|}
\cline { 2 - 4 } \multicolumn{1}{c|}{} & $\begin{array}{c}\text { Alfa } \\
\text { Cronbach }\end{array}$ & $\begin{array}{c}\text { Fiabilidad } \\
\text { compuesta }\end{array}$ & $\begin{array}{c}\text { Dijkstra y } \\
\text { Henseler }\end{array}$ \\
\hline Valores (fiabilidad si $>0,7)$ & 0,719 & 0,721 & 0,723 \\
\hline
\end{tabular}

Validez convergente (convergencia de un constructo en explicar sus indicadores): medida por la Varianza Extraída Media (AVE). En el constructo "Capacidad de Adaptación" AVE = 0,564 >0,5.

Validez discriminante (grado en el que cada constructo es diferente de los demás del modelo): evaluación por dos criterios.

- Criterio de Fornell-Larcker: varianza capturada por un constructo de sus indicadores debe ser mayor que la varianza compartida entre él y los demás del modelo (Fornell \& Larcker, 1981).

Tabla 8. Criterio de Fornell-Larcker

\begin{tabular}{|c|c|}
\cline { 2 - 2 } \multicolumn{1}{c|}{} & Adaptación \\
\hline Adaptación & $\mathbf{0 , 7 5 1}$ \\
\hline TIC & 0,699 \\
\hline Innovación & 0,682 \\
\hline Flexibilidad & 0,587 \\
\hline
\end{tabular}

- Análisis de las cargas cruzadas: las cargas factoriales de los indicadores deben tener mayor valor con su propia variable que con las demás.

Tabla 9. Cargas cruzadas

\begin{tabular}{|c|c|c|c|c|}
\cline { 2 - 5 } \multicolumn{1}{c|}{} & Adaptación & TIC & Innovación & Flexibilidad \\
\hline Org & $\mathbf{0 , 7 1 9}$ & 0,369 & 0,488 & 0,535 \\
\hline Rel.Cl & $\mathbf{0 , 7 8 2}$ & 0,508 & 0,535 & 0,516 \\
\hline Dig & 0,587 & $\mathbf{1}$ & 0,547 & 0,147 \\
\hline IDi & 0,682 & 0,547 & $\mathbf{1}$ & 0,312 \\
\hline Aut & 0,572 & 0,116 & 0,307 & $\mathbf{0 , 8 5 4}$ \\
\hline Multi & 0,649 & 0,14 & 0,246 & $\mathbf{0 , 8 9 8}$ \\
\hline
\end{tabular}

Los resultados validan todos los modelos de medida formativos y reflexivo del modelo teórico depurado.

\subsubsection{Evaluación del modelo estructural}

Los efectos estimados, directos e indirectos, entre las variables exógenas y las endógenas fueron los siguientes: 
Tabla 10. Efectos directos (coeficientes path), indirectos y totales

\begin{tabular}{|l|c|c|c|}
\cline { 2 - 4 } \multicolumn{1}{c|}{} & $\begin{array}{c}\text { Efectos } \\
\text { directos }\end{array}$ & $\begin{array}{c}\text { Efectos } \\
\text { indirectos }\end{array}$ & Efectos totales \\
\hline Flexibilidad -> Adaptación & 0.547 & 0.104 & 0.651 \\
\hline Flexibilidad -> Innovación & 0.312 & & \\
\hline Innovación -> Adaptación & 0.335 & & \\
\hline TIC -> Adaptación & 0.324 & & \\
\hline
\end{tabular}

Para la validación del modelo estructural se han considerado (Hair et al., 2019): problemas de colinealidad, valores de los coeficientes path, coeficiente de determinación $\left(\mathrm{R}^{2}\right)$, tamaño de los efectos $\left(\mathrm{f}^{2}\right)$ y relevancia predictiva.

Análisis de la presencia de problemas de colinealidad: para asegurar que no existe multicolinealidad entre las variables precedentes de cada constructo endógeno, el Factor de Inflación de la Varianza (VIF) ha de ser menor que 3.

\section{Tabla 11. VIF del modelo estructural}

\begin{tabular}{|l|c|c|}
\cline { 2 - 3 } \multicolumn{1}{c|}{} & Adaptación & Innovación \\
\hline Flexibilidad & 1,109 & 1 \\
\hline Innovación & 1,547 & \\
\hline Desarrollo TIC & 1,427 & \\
\hline
\end{tabular}

Evaluación del signo algebraico, magnitud y significación estadística de los coeficientes path (relaciones hipotetizadas entre las variables), a partir de los datos de la figura 3.

-Signo y magnitud de los coeficientes: todos los coeficientes son de signo positivo, conforme a las relaciones positivas hipotetizadas. Muestran magnitudes de efectos medias o moderadas.

-Valoración de la significación de los efectos: mediante la técnica de remuestreo bootstrapping se ha comprobado el nivel de significación de cada coeficiente $(\mathrm{p}<0,05)$.

\section{Tabla 12. Resultados bootstrapping efectos}

\begin{tabular}{|l|c|c|c|c|c|}
\cline { 2 - 6 } \multicolumn{1}{l|}{} & $\begin{array}{c}\text { Muestra } \\
\text { original }\end{array}$ & $\begin{array}{c}\text { Media } \\
\text { de la } \\
\text { muestra }\end{array}$ & $\begin{array}{c}\text { Desviación } \\
\text { estándar }\end{array}$ & Estadísticos t & P Valores \\
\hline $\begin{array}{l}\text { Flexibilidad -> } \\
\text { Adaptación }\end{array}$ & 0,547 & 0,548 & 0,106 & 5,175 & $\mathbf{0}$ \\
\hline $\begin{array}{l}\text { Flexibilidad -> } \\
\text { Innovación }\end{array}$ & 0,312 & 0,324 & 0,112 & 2,797 & $\mathbf{0 , 0 0 3}$ \\
\hline $\begin{array}{l}\text { Innovación -> } \\
\text { Adaptación }\end{array}$ & 0,335 & 0,336 & 0,111 & 3,014 & 0,001 \\
\hline TIC -> Adaptación & 0,324 & 0,323 & 0,097 & 3,345 & $\mathbf{0}$ \\
\hline $\begin{array}{l}\text { Flexibilidad -> Innova- } \\
\text { ción -> Adaptación }\end{array}$ & 0,105 & 0,112 & 0,059 & 1,769 & $\mathbf{0 , 0 3 9}$ \\
\hline
\end{tabular}


Los resultados muestran que las cuatro hipótesis formuladas constituyen relaciones significativas.

Valoración del coeficiente de determinación (R2): los valores de obtenidos de $\mathrm{R}^{2} \mathrm{y}$ de $\mathrm{R}^{2}$ ajustado para la variable endógena “Adaptación” han sido de 0,801 y 0,792 respectivamente, lo que indica un poder explicativo sustancial (Chin, 1998). predictoras:

Respecto a la varianza explicada de "Adaptación" por cada una de sus variables

Tabla 13. Varianza explicada por cada predictor

\begin{tabular}{|l|c|c|c|}
\cline { 2 - 4 } \multicolumn{1}{c|}{} & Coef. path & Correlación & $\begin{array}{c}\text { Varianza } \\
\text { explicada }\end{array}$ \\
\hline Flexibilidad & 0,547 & 0,699 & $\mathbf{0 , 3 8 2 3}$ \\
\hline Innovación & 0,335 & 0,682 & $\mathbf{0 , 2 2 8 4}$ \\
\hline TIC & 0,324 & 0,587 & $\mathbf{0 , 1 9 0 1}$ \\
\hline
\end{tabular}

Valoración de los tamaños de los efectos ( $f 2)$ : todos los efectos observados son de tamaño grande (Cohen, 1988).

Tabla 14. Tamaño de los efectos

\begin{tabular}{|l|c|}
\cline { 2 - 2 } \multicolumn{1}{c|}{} & $\mathbf{f}^{2}$ \\
\hline Flexibilidad & 1,352 \\
\hline Innovación & 0,363 \\
\hline TIC & 0,369 \\
\hline
\end{tabular}

Valoración de la relevancia predictiva: se empleó el valor $\mathrm{Q}^{2}$ del test de StoneGeisser como medida del poder predictivo del modelo fuera de la muestra utilizada. Para su cálculo, se ha empleado el procedimiento blindfolding, reutilizando la muestra omitiendo cada d-ésimo dato del constructo endógeno, estimando los distintos parámetros con los datos restantes, y prediciendo a partir de ellos los valores omitidos (Chin, 1998). El procedimiento se ha realizado con una distancia de omisión $\mathrm{D}=7^{1}$. El valor de $\mathrm{Q}^{2}$ se calculó mediante el enfoque de redundancia validada de forma cruzada (estimaciones tanto del modelo estructural como de medida para la predicción).

\section{Tabla 15. Resultados blindfolding-Redundancia de constructo validada de forma cruzada}

\begin{tabular}{|l|c|c|c|}
\cline { 2 - 4 } \multicolumn{1}{c|}{} & $\begin{array}{c}\text { Suma de las } \\
\text { observaciones al } \\
\text { cuadrado (SSO) }\end{array}$ & $\begin{array}{c}\text { Suma de los errores } \\
\text { de predicción al } \\
\text { cuadrado (SSE) }\end{array}$ & $\mathbf{Q}^{2}=1$-SSE/SSO \\
\hline Adaptación & 152 & 85,456 & 0,438 \\
\hline Innovación & 76 & 72,035 & 0,052 \\
\hline
\end{tabular}

1 Los valores recomendados para D son entre 5 y 10 (Hair et al., 2019). Además, el cociente entre el tamaño muestral y la distancia D no puede ser un número entero, para evitar que en la matriz de datos se elimine siempre el mismo conjunto de observaciones en cada ronda. En este caso, 76/7. 
El valor obtenido, mayor que 0, refleja relevancia predictiva. En el caso de "Capacidad de adaptación", al situarse entre 0,25 y 0,5 indica relevancia de tamaño medio y en el de "Innovación", baja (Hair et al., 2019b).

\subsubsection{Ajuste global del modelo}

Mediante la evaluación del grado de discrepancia entre la matriz de correlaciones del modelo estimado y la matriz de correlaciones empírica. Se utilizó el SRMR, Standardized Root Mean Square residual, como reflejo de dicha divergencia. SRMR ha de ser menor que 0,08 (Hu \& Bentler, 1998), y a menor valor de SRMR, mejor ajuste. En nuestro modelo, $\mathrm{SRMR}=0,026$.

\section{Conclusiones y discusión}

La presente investigación ha explorado si determinados factores transversales a las organizaciones pueden facilitar su adaptación al entorno competitivo resultante de la pandemia de COVID-19. Se han encontrado relaciones significativas entre tres características organizacionales y la capacidad de adaptación al nuevo escenario: flexibilidad laboral, desarrollo de TIC y esfuerzo en innovación. Este último ejerce además un papel mediador en la relación entre flexibilidad y capacidad de adaptación, incrementándose el efecto total de la primera sobre la segunda. Los resultados obtenidos han validado las cuatro hipótesis formuladas.

Respecto a la capacidad de adaptación, se ha encontrado que las variables más adecuadas para su observación son el nivel de incidencias sobre la organización y monitorización del trabajo y sobre las relaciones con los clientes. Aspectos como los ajustes de plantilla, cambios en el mix de la oferta o recortes estructurales no han mostrado ser elementos cuyo factor común principal sea la capacidad de adaptación, debiéndose para ellos explorar otros factores internos y externos de la empresa- como la estrategia genérica, las particularidades de su cadena de suministro, limitaciones regulatorias específicas, estacionalidades del sector, etc.

Las principales contribuciones de la investigación son, en primer lugar, la delimitación de determinantes que parecen favorecer la capacidad de adaptación de las empresas al entorno competitivo consecuencia de la COVID-19. Si bien todas las relaciones hipotetizadas han resultado significativas, el efecto mayor se da en la influencia positiva que ejerce la flexibilidad laboral funcional, lo que está en línea con trabajos precedentes que muestran que prácticas como la descentralización o la polivalencia favorecen la competitividad de las organizaciones (Bloom et al., 2010; Sánchez et al., 2011). En segundo lugar, los resultados obtenidos pueden orientar a focalizar esfuerzos. Muchos de los efectos provocados por la crisis van a permanecer en el largo plazo (Gruszczynski, 2020; Ortega-Vivanco, 2020), constituyéndose la COVID-19 en catalizador de tendencias observadas desde hace años. Así actuaciones hacia la digitalización que permitan actividades como la monitorización en tiempo real, el teletrabajo o desarrollar canales online con clientes y proveedores; la polivalencia de los trabajadores que favorece la adaptación continua a la demanda, mayor motivación y espíritu de trabajo en equipo; o los esfuerzos en innovar, que acarrean consecuencias organizacionales de excelencia a lo largo de toda la organización más allá de los $J$ innovadores pasan a convertirse en un must para las empresas en el corto y medio plazo.

Los resultados obtenidos y conclusiones extraídas están en línea con diversas investigaciones previas. Siguiendo a Milgrom y Roberts (1992), puede concluirse que el desarrollo de las TIC permite gestionar de forma más eficiente los problemas de información y de coordinación de una organización, lo cual incide directamente en su 
adaptación al entorno competitivo. En el contexto actual, Almeida et al. (2020) resaltan la importancia del papel que la digitalización puede ejercer en la competitividad de las empresas en la era pos-COVID-19. Y Fields et al. (2020) observan que las prácticas de industria 4.0 han ayudado a mantener las actividades empresariales durante la crisis.

Además del papel moderador y mediador que ejerce la innovación, se ha observado que los efectos de la pandemia han ejercido un papel catalizador sobre la innovación (Heinonen \& Strandvik, 2020), y que en el entorno actual la capacidad de innovación influye significativamente en el éxito empresarial (Putra et al., 2020).

Como próximos pasos a seguir en la investigación queda profundizar en las relaciones exploradas y analizar su evolución temporal mediante el uso de datos de panel, explorando además la relación existente entre las variables consideradas y diversos indicadores de rendimiento organizacional, incorporando en paralelo otras variables en el estudio, como la cooperación entre empresas como fuente de desarrollo económico local en momentos de crisis (Carpio, 2020; Vergara-Romero \& Sorhegui-Ortega, 2020). Así mismo, también aumentar el tamaño muestral para avanzar en el camino del estudio confirmatorio de las relaciones entre las variables. Finalmente, analizar el papel moderador que variables como el tipo de propiedad o el tamaño pueden ejercer sobre las relaciones analizadas.

\section{Referencias}

Alday, M.Á., Amigo, M.C., \& Heras, H.A. (2020). Estructuras organizativas para organizaciones resilientes. Una reflexión desde el punto de vista del diseño organizativo. Boletín de Estudios Económicos, 75(231), 475-500.

Almeida, F., Santos, J.D., \& Monteiro, J.A. (2020). The challenges and opportunities in the digitalization of companies in a post-COVID-19 World. IEEE Engineering Management Review, 48(3), 97-103. https://doi.org/10.1109/EMR.2020.3013206

Atkinson, J. (1984). Manpower strategies for flexible organisations. Personnel management, 16(8), 28-31.

Barney, J. B. (2001). Resource-based theories of competitive advantage: A ten-year retrospective on the resource-based view. Journal of Management, 27(6), 643-650. https://doi.org/10.1177/014920630102700602

BDE (2020). Informe trimestral de la economía española. Boletín Económico/Banco de España [Artículos], n. 4.

Bharadwaj, A.S. (2000). A resource-based perspective on information technology capability and firm performance: an empirical investigation. MIS Quarterly, 169-196. https://doi.org/10.2307/3250983

Blanco, R., Mayordomo, S., Menéndez, Á., \& Menéndez, Á. (2020). El impacto de la crisis del Covid-19 sobre la situación financiera de las empresas no financieras en 2020: evidencia basada en la Central de Balances. Boletín Económico, (4/2020), 1-23.

Bloom, N., \& Van Reenen, J. (2007). Measuring and explaining management practices across firms and countries. The Quarterly Journal of Economics, 122(4), 1351-1408. https://doi.org/10.1162/qjec.2007.122.4.1351

Bloom, N., Sadun, R., \& Van Reenen, J. (2010). Recent advances in the empirics of organizational economics. Annual Review of Economics, 2(1), 105-137. https://doi.org/10.1146/annurev.economics.050708.143328

Bloom, N., Garicano, L., Sadun, R., \& Van Reenen, J. (2014). The distinct effects of information technology and communication technology on firm organization. Management Science, 60(12), 2859-2885. https://doi.org/10.1287/mnsc.2014.2013

Bueno, E. (2007). Organización de empresas. Estructura, procesos y modelos. Pirámide.

Carpio, S. (2020). La cooperación entre firmas en el desarrollo regional: una revisión. Retos. Revista de Ciencias de la Administración y Economía, 10(19), 117-133. https://doi.org/10.17163/ret.n19.2020.07

Chin, W.W. (1998). The partial least squares approach to structural equation modeling. Modern Methods for Business Research, 295(2), 295-336. 
Chin, W.W. (2010). How to write up and report PLS analyses. In Handbook of partial least squares (pp. 655-690). Springer, Berlin, Heidelberg.

Cobo-Romaní, J. C. (2009). El concepto de tecnologías de la información. Benchmarking sobre las definiciones de las TIC en la sociedad del conocimiento. ZER-Revista de Estudios de Comunicación, 14(27), 295-318. https://bit.ly/2NoSUrL

Crick, J.M., \& Crick, D. (2020). Coopetition and COVID-19: Collaborative business-to-business marketing strategies in a pandemic crisis. Industrial Marketing Management, 88, 206-213. https:// doi.org/10.1016/j.indmarman.2020.05.016

Diamantopoulos, A., \& Siguaw, J.A. (2006). Formative versus reflective indicators in organizational measure development: A comparison and empirical illustration. British Journal of Management, 17(4), 263-282. https://doi.org/10.1111/j.1467-8551.2006.00500.x

Dijkstra, T.K., \& Henseler, J. (2015). Consistent partial least squares path modeling. MIS Quarterly, 39(2). https://doi.org/10.2307/26628355

Draca, M., Sadun, R., \& Van Reenen, J. (2007). Productivity and ICTs: a review of the evidence. In The Oxford Handbook of Information and Communication Technologies (pp. 100-147). Oxford University Press.

Duchek, S. (2020). Organizational resilience: a capability-based conceptualization. Business Research, 13(1), 215-246. https://doi.org/10.1007/s40685-019-0085-7

Fields, Z., Abdullah, Z.M., Musisi, A.N., \& Mitchley, N.K. (2020). Using collective creativity and industry 4.0 technology to reduce the negative impact of a pandemic on entrepreneurs. In Handbook of Research on Using Global Collective Intelligence and Creativity to Solve Wicked Problems (pp. 133-155). IGI Global. https://doi.org/10.4018/978-1-7998-2385-8.ch007

Fornell, C., \& Larcker, D.F. (1981). Evaluating structural equation models with unobservable variables and measurement error. Journal of Marketing Research, 18(1), 39-50. https://doi.org/10.1177/002224378101800104

Freije, I., \& Aláez, M. (2020). Resiliencia estratégica: ¿una capacidad estratégica distintiva o umbral? Boletín de Estudios Económicos, 75(231), 453-474.

Garamendi, A. (2020). La economía española y su tejido productivo: la resiliencia, el valor para superar esta crisis y mirar el futuro. Boletín de Estudios Económicos, 75(231), 433-451.

Grant, R.M. (2014). Dirección estratégica. Thomson-Civitas.

Gruszczynski, L. (2020). The COVID-19 pandemic and international trade: Temporary turbulence or paradigm shift? European Journal of Risk Regulation, 11(2), 337-342. https://doi.org/10.1017/err.2020.29

Guderian, C.C., Bican, P.M., Riar, F.J., \& Chattopadhyay, S. (2020). Innovation management in crisis: patent analytics as a response to the COVID囚19 pandemic. REDD Management. https://doi.org/10.1111/radm.12447

Hair, J.F., Hult, G.T.M., Ringle, C.M., Sarstedt, M., Castillo-Apraiz, J., Cepeda-Carrión, G., \& Roldán, J.L. (2019a). Manual de partial least squares structural equation modeling (pls-sem). OmniaScience Scholar.

Hair, J.F., Risher, J.J., Sarstedt, M., \& Ringle, C.M. (2019b). When to use and how to report the results of PLS-SEM. European Business Review. https://doi.org/10.1108/EBR-11-2018-0203

Hax, A.C., \& Majluf, N.S. (2015). Estrategia para el liderazgo competitivo. De la visión a los resultados. Prentice-Hall.

Heinonen, K., \& Strandvik, T. (2020). Reframing service innovation: COVID-19 as a catalyst for imposed service innovation. Journal of Service Management.

Hermann, C.F. (1963). Some consequences of crisis which limit the viability of organizations. Administrative Science Quarterly, 61-82. https://doi.org/10.2307/2390887

Hu, L.T., \& Bentler, P.M. (1998). Fit indices in covariance structure modeling: Sensitivity to underparameterized model misspecification. Psychological Methods, 3(4), 424. https://doi.org/10.1037/1082-989X.3.4.424 
Lee, S.M., \& Trimi, S. (2020). Convergence innovation in the digital age and in the COVID-19 pandemic crisis. Journal of Business Research, 123, 14-22. https://doi.org/10.1016/j.jbusres.2020.09.041

Lengnick-Hall, C.A., Beck, T.E., \& Lengnick-Hall, M.L. (2011). Developing a capacity for organizational resilience through strategic human resource management. Human Resource Management Review, 21(3), 243-255. https://doi.org/10.1016/j.hrmr.2010.07.001

López-Mielgo, N., Montes-Peón, J.M., \& Vázquez-Ordás, C. (2012). ¿Qué necesita una empresa para innovar? Investigación, experiencia y persistencia. Revista Europea de Dirección y Economía de la Empresa, 21(3), 266-281. https://doi.org/10.1016/j.redee.2012.05.005

Milgrom, P., \& Roberts, J. (1992): Economics, Organization and Management. Prentice Hall, Inc.

Observatorio Nacional de las Telecomunicaciones y de la Sociedad de la Información (2011). Informe e-pyme 2010: Análisis sectorial de la implantación de las TIC en la PYME española. Ministerio de Industria, Comercio y Turismo del Gobierno de España. https://bit.ly/3aPkV4v

Observatorio Nacional de las Telecomunicaciones y de la Sociedad de la Información (2020). Informe e-pyme 2019: Análisis sectorial de la implantación de las TIC en las empresas españolas. Madrid: ONTSI. http://doi.org/10.30923/2341-4030-2020

OECD (2021). OECD Economic Outlook No 108 (Edition 2020/2), OECD Economic Outlook: Statistics and Projections (database). https://doi.org/10.1787/c59fcffd-en

Ortega-Vivanco, M. (2020). Efectos del Covid-19 en el comportamiento del consumidor: Caso Ecuador.RETOS. Revista de Ciencias de la Administración y Economía, 10(20), 233-247. https://orcid.org/0000-0003-4647-4343

Pearson, C.M., \& Clair, J.A. (1998). Reframing crisis management. Academy of Management Review, 23(1), 59-76. https://doi.org/10.5465/amr.1998.192960

Prajogo, D.I., \& Ahmed, P.K. (2006). Relationships between innovation stimulus, innovation capacity, and innovation performance. RED Management, 36(5), 499-515. https://doi.org/10.1111/j.1467-9310.2006.00450.x

Putra, I., Sunarsih, N., Novitasari, L., \& Setini, M. (2020). Exploring the relationship between social capital, innovation capability and innovation during the coronavirus pandemic. Uncertain Supply Chain Management, 8(4). https://doi.org/857-864. 10.5267/j.uscm.2020.5.007

Ravichandran, T. \& Lertwongsatien, C. (2005). Effect of information systems resources and capabilities on firm performance: A resource-based perspective. Journal of Management Information Systems, 21(4), 237-276. https://doi.org/10.1080/07421222.2005.11045820

Sánchez, Á.M., Jiménez, M.J.V., \& Pérez, M.P. (2011). Innovación y flexibilidad de recursos humanos: el efecto moderador del dinamismo del entorno. Revista Europea de Dirección y Economía de la Empresa, 20(1), 41-68.

Schaltegger, S. (2020). Sustainability learnings from the COVID-19 crisis. Opportunities for resilient industry and business development. Sustainability Accounting, Management and Policy Journal. https://doi.org/10.1108/SAMPJ-08-2020-0296

Sharma, H.B., Vanapalli, K.R., Cheela, V.S., Ranjan, V.P., Jaglan, A.K., Dubey, B., \& Bhattacharya, J. (2020). Challenges, opportunities, and innovations for effective solid waste management during and post COVID-19 pandemic. Resources, Conservation and Recycling, 162, 105052. https://doi.org/10.1016/j.resconrec.2020.105052

Smart, C., \& Vertinsky, I. (1984). Strategy and the environment: A study of corporate responses to crises. Strategic Management Journal, 5(3), 199-213. https://doi.org/10.1002/smj.4250050302

Volberda, H.W. (1996). Toward the flexible form: how to remain vital in hypercompetitive environments. Organization Science, 7(4), 359-374. https://doi.org/10.1002/smj.4250050302

Taleb, N.N. (2008). El cisne negro: el impacto de lo altamente improbable. Paidós.

Thompson, A. \& Strickland, A. (2004). Administración estratégica. MacGraw Hill.

Vergara-Romero, A., \& Sorhegui-Ortega, R. (2020). Factores de desarrollo local desde el enfoque de la gestión organizativa Revista de la Universidad del Zulia, 11(31), 86-96.

https://doi.org/10.46925//rdluz.31.07

Weick, K.E., \& Sutcliffe, K.M. (2001). Managing the unexpected (Vol. 9). Jossey-Bass. 
Werts, C.E., Linn, R.L., \& Jöreskog, K.G. (1974). Interclass reliability estimates: testing structural assumptions. Educational and Psychological Measurement, 34, 25-33. https://doi.org/10.1177/001316447403400104

Wright, P.M., \& Snell, S.A. (1998). Toward a unifying framework for exploring fit and flexibility in strategic human resource management. Academy of Management Review, 23(4), 756-772. https://doi.org/10.5465/amr.1998.1255637 